\title{
AN ELEMENTARY PROOF THAT RATIONALLY ISOMETRIC QUADRATIC FORMS ARE ISOMETRIC
}

\author{
URIYA A. FIRST
}

\begin{abstract}
Let $R$ be a valuation ring with fraction field $K$ and $2 \in R^{\times}$. We give an elementary proof of the following known result: Two unimodular quadratic forms over $R$ are isometric over $K$ if and only if they are isometric over $R$. Our proof does not use cancelation of quadratic forms and yields an explicit algorithm to construct an isometry over $R$ from a given isometry over $K$. The statement actually holds for hermitian forms over valuated involutary division rings, provided mild assumptions.
\end{abstract}

Let $S / R$ be a separable commutative ring extension with $R$ a local integral domain, let $F$ be the fraction field of $R$, and let $A$ be an Azumaya $S$-algebra admitting an involution $\sigma$ with $R=S^{\sigma}:=\left\{s \in S: s^{\sigma}=s\right\}$. Assume that $2 \in R^{\times}$. It was shown in 4 that when $R$ is regular and contains a field, a unimodular hermitian form over $A$ is hyperbolic over $A \otimes_{R} F$ if and only if it is hyperbolic over $A$. This is a special case of the Grothendieck-Serre conjecture; see for instance [?, §1] and related papers. The same statement was established in [1, Pr. 3.6] when $R$ is a valuation ring (or even an intersection of finitely many) and $A$ has no zero-divisors. By invoking a cancelation theorem of Keller ([3, Th. 3.4.2]) as done in [2, Pr. 2.14], this implies that when $R$ is a valuation ring or local regular ring containing a field, two unimodular hermitian forms over $(A, \sigma)$ are isometric over $A \otimes_{R} F$ if and only if they are isometric over $R$. Special cases of these results were proved much earlier by various authors (e.g. see [6, $\S 6.2]$ and similar references).

We give an elementary proof of the following special case:

Theorem 1. Let $K / F$ be a field extension admitting an involution $\sigma \in \operatorname{Gal}(K / F)$ such that $K^{\sigma}=F$, and let $\nu: K^{\times} \rightarrow \Gamma$ be an (additive) valuation with $\nu \circ \sigma=\nu$ and $\nu(2)=0$. Denote by $S$ and $R$ the valuation rings of $\nu$ in $K$ and $F$, respectively. If $K / F$ is unramified with respect to $\nu$ (i.e. $\operatorname{im}\left(\left.\nu\right|_{F}\right)=\Gamma$ ), then two unimodular 1-hermitian forms over $(S, \sigma)$ are isometric over $K \cong S \otimes_{R} F$ if and only if they are isometric over $S$.

Our proof is elementary and avoids cancelation of hermitian forms over local rings. Furthermore, it yields an explicit algorithm to construct an isometry over $S$ from an isometry over $K$. (The basic operations required for the algorithm are arithmetic operations in $K$ and $\Gamma$, applying $\nu$, and producing elements of $F$ with a given valuation.)

Before giving the proof, let us recall some of the definitions; see [6] for an extensive discussion: Let $\varepsilon= \pm 1$. An $\varepsilon$-hermitian space over a ring with involution $(S, \sigma)$ consists of a pair $(M, h)$ where $M$ is a projective left $S$-module, and $h: M \times M \rightarrow S$

Date: February 17, 2018.

2010 Mathematics Subject Classification. 11E08.

Key words and phrases. quadratic form, hermitian form, valuation, rational isomorphism, Grothendieck-Serre conjecture.

This research was supported by a Swiss National Foundation of Science Grant no. IZK0Z2_151061. 
is a biadditive map satisfying $h(a x, b y)=a h(x, y) b^{\sigma}$ and $h(x, y)=\varepsilon h(y, x)^{\sigma}$ for all $x, y \in M$ and $a, b \in M$. In this case, $h$ is called an $\varepsilon$-hermitian form. We say that $h$ is unimodular if the map $\tilde{h}: M \rightarrow \operatorname{Hom}_{S}(M, S)$ given by $\tilde{h}(x)=[y \mapsto h(y, x)]$ is an isomorphism. Two hermitian spaces $(M, h)$ and $\left(M^{\prime}, h^{\prime}\right)$ are called isometric if there is an isomorphism $u: M \rightarrow M^{\prime}$ such that $h^{\prime}(u x, u y)=h(x, y)$. If $(K, \tau)$ is a ring with involution containing $(S, \sigma)$, then $(M, h)$ gives rise to an $\varepsilon$-hermitian space $\left(M_{K}, h_{K}\right)$ over $(K, \tau)$ defined by $M_{K}=K \otimes_{S} M$ and $h_{K}(a \otimes x, b \otimes y)=a h(x, y) b^{\tau}$ $(x, y \in M, a, b \in K)$. We say that $(M, h)$ and $\left(M^{\prime}, h^{\prime}\right)$ are isometric over $(K, \tau)$ if $\left(M_{K}, h_{K}\right)$ and $\left(M_{K}^{\prime}, h_{K}^{\prime}\right)$ are isometric.

Proof of Thoerem 1. For a matrix $a=\left(a_{i j}\right)_{i, j}$ over $K$, write $a^{*}:=\left(a_{j i}^{\sigma}\right)_{i, j}$. We say that $a$ is $*$-symmetric if $a^{*}=a$.

Step 0. Assume $(M, h)$ and $\left(M^{\prime}, h^{\prime}\right)$ are unimodular 1-hermitian spaces over $S$ that are isometric over $K$. Then $\operatorname{dim}_{S} M=\operatorname{dim}_{S} M^{\prime}$, hence we may assume $M=M^{\prime}=$ $S^{n}$ (viewed as row vectors). In this case, there are unique $*$-symmetric matrices $a, b \in \mathrm{GL}_{n}(S)$ such that $h(u, v)=u a v^{*}$ and $h^{\prime}(u, v)=u b v^{*}$ for all $u, v \in S^{n}$. It is well-known that $h \cong h^{\prime}$ over $K$ (resp. $S$ ) if and only if there exists $u \in \operatorname{GL}_{n}(K)$ (resp. $u \in \mathrm{GL}_{n}(S)$ ) such that $u a u^{*}=b$. It is thus enough to show that if $u a u^{*}=b$ with $u \in \mathrm{GL}_{n}(K)$, then $u$ can be taken to be in $\operatorname{GL}_{n}(S)$.

Step 1. We may assume $u$ is diagonal. Indeed, it is a standard claim that in every Bézout domain (i.e. a domain whose f.g. ideals are principle), $S$ in particular,

$$
\operatorname{GL}_{n}(K)=\operatorname{GL}_{n}(S) \cdot T \cdot \mathrm{GL}_{n}(S),
$$

where $T$ denotes the diagonal matrices in $\mathrm{GL}_{n}(K)$. For the sake of completeness, we shall recall the proof for valuation rings in Proposition 2 Now, using (1), write $u=x u^{\prime} y$ with $x, y \in \operatorname{GL}_{n}(S)$ and $u^{\prime} \in T$. We may replace $a, b, u$ with yay* $x^{-1} b\left(x^{-1}\right)^{*}, u^{\prime}$.

Step 2. Let $I_{r}$ denote the identity matrix of size $r$. We claim that we may assume $u$ is of the form $\pi I_{r} \oplus \pi^{-1} I_{r} \oplus I_{n-2 r}$ for some $\pi \in \operatorname{Jac}(R)$ and $r>0$.

For a matrix $x=\left(x_{i j}\right)_{i, j}$, write $\nu(x)=\left(\nu\left(x_{i j}\right)\right)_{i, j}$. Then $\nu(x)$ is a matrix with entries in $\Gamma \cup\{\infty\}$ (where $\nu(0)=\infty$ ). Let $u_{1}, \ldots, u_{n}$ be the diagonal entries of $u$ and let $\gamma_{t}>\gamma_{t-1}>\cdots>\gamma_{1}>\gamma_{0}=0$ be the absolute values of the valuations of $u_{1}, \ldots, u_{n}$ together with $0 \in \Gamma$. Conjugating $a, b, u$ by a suitable permutation matrix, we may assume that

$$
\nu\left(u_{1}\right)=\cdots=\nu\left(u_{r}\right)=\gamma_{t}, \quad \nu\left(u_{r+1}\right)=\cdots=\nu\left(u_{r+s}\right)=-\gamma_{t},
$$

and $\left|\nu\left(u_{i}\right)\right|<\gamma_{t}$ for all $i>r+s$.

We claim that $r=s$. Indeed, write $\left(\alpha_{i j}\right)=\nu(a),\left(\beta_{i j}\right)=\nu(b)$ and $\tau_{i}=\nu\left(u_{i}\right)=$ $\nu\left(u_{i}^{\sigma}\right)$. Then

$$
\left(\beta_{i j}\right)_{i, j}=\nu\left(u a u^{*}\right)=\left(\alpha_{i j}+\tau_{i}+\tau_{j}\right)_{i, j},
$$

hence $\alpha_{i j}+\tau_{i}+\tau_{j} \geq 0$ for all $i, j$. Since $\tau_{i}+\tau_{j}<0$ when $r<i \leq r+s$ and $r<j$, we have

$$
\nu\left(a_{i j}\right)>0 \quad \forall \quad r<i \leq r+s, \quad r<j .
$$

If $s>r$, this implies that rows $r+1, \ldots, r+s$ of $a$ are linearly dependant over $S / \operatorname{Jac}(S)$, contrary to our assumption that $a$ is invertible over $S$. Thus, $s \leq r$. Applying a similar argument to show that $\nu\left(\beta_{i j}\right)>0$ when $i \leq r$ and $j \leq r$ or $r+s<j$ yields that $s \geq r$, so $s=r$.

We now apply induction to $t$, the case $t=0$ being clear. Pick an element $\pi \in R$ of valuation $\gamma_{t}-\gamma_{t-1}$ (here we use the assumption that $K / F$ is unramified), let $u^{\prime}=\pi I_{r} \oplus \pi^{-1} I_{r} \oplus I_{n-2 r}$, and write $b^{\prime}=u^{\prime} a u^{\prime *}$ and $\left(\beta_{i j}^{\prime}\right)=\nu\left(u^{\prime} a u^{\prime *}\right)$. We claim 
that $\beta_{i j}^{\prime} \geq 0$ for all $i$. As $b^{\prime}$ is $*$-symmetric, we only need to verify this for $i \leq j$. Indeed, we have

$$
\beta_{i j}^{\prime}= \begin{cases}\alpha_{i j}+2 \gamma_{t}-2 \gamma_{t-1} & i \leq j \leq r \\ \alpha_{i j}-2 \gamma_{t}+2 \gamma_{t-1} & r<i \leq j \leq 2 r \\ \alpha_{i j} & i \leq r<j \leq 2 r \text { or } 2 r<i \leq j \\ \alpha_{i j}+\gamma_{t}-\gamma_{t-1} & i \leq r<2 r<j \\ \alpha_{i j}-\gamma_{t}+\gamma_{t-1} & r<i \leq 2 r<j\end{cases}
$$

All the cases are clear except the second and the fifth. Recall that $\alpha_{i j}+\tau_{i}+\tau_{j}=\beta_{i j}$. Now, in second case, we have

$$
\alpha_{i j}-2 \gamma_{t}+2 \gamma_{t-1}=\alpha_{i j}+\tau_{i}+\tau_{j}+2 \gamma_{t-1}=\beta_{i j}+2 \gamma_{t-1} \geq 0,
$$

and in the fifth case, writing $\left|\tau_{j}\right|=\gamma_{s}$ for $s<t$ (and noting that $\tau_{i}=-\gamma_{t}$ ), we get

$$
\alpha_{i j}-\gamma_{t}+\gamma_{t-1}=\beta_{i j}-\tau_{j}+\gamma_{t-1} \geq \beta_{i j}+\gamma_{t-1}-\gamma_{s} \geq 0
$$

as required. Thus, $b^{\prime} \in \mathrm{M}_{n}(S)$. Since $\nu\left(\operatorname{det}\left(b^{\prime}\right)\right)=\nu\left(\operatorname{det}\left(u^{\prime} a u^{\prime *}\right)\right)=\nu(\operatorname{det}(a))=0$ (because $\operatorname{det}\left(u^{\prime}\right)=1$ ), we have $b^{\prime} \in \mathrm{GL}_{n}(S)$. Therefore, we may replace $a, b, u$ with $a, b^{\prime}, u^{\prime}$ and apply induction to $b^{\prime}, b, u u^{\prime-1}$ (the parameter $t$ is decreased by 1 ).

Step 3. We may assume $n=2 r$ and $u=\pi I_{r} \oplus \pi^{-1} I_{r}$ with $\pi \in \operatorname{Jac}(R)$. Indeed, suppose $u=\pi I_{r} \oplus \pi^{-1} I_{r} \oplus I_{n-2 r}$ as in step 2 . Then we may write

$$
a=\left[\begin{array}{ccc}
a_{11} & a_{12} & a_{13} \\
a_{12}^{*} & \pi^{2} a_{22} & \pi a_{23} \\
a_{13}^{*} & a_{23}^{*} \pi & a_{33}
\end{array}\right], \quad b=\left[\begin{array}{ccc}
\pi^{2} a_{11} & a_{12} & \pi a_{13} \\
a_{12}^{*} & a_{22} & a_{23} \\
a_{13}^{*} \pi & a_{23}^{*} & a_{33}
\end{array}\right]
$$

where $a_{11}, a_{12}, a_{13}, a_{22}, a_{23}, a_{33}$ are matrices over $S$ and $a_{11}, a_{12}, a_{22}$ are of size $r \times r$. Observe that the image of $a$ in $\mathrm{M}_{n}(S / \operatorname{Jac}(S))$ has the form

$$
\left[\begin{array}{lll}
* & * & * \\
* & 0 & 0 \\
* & 0 & *
\end{array}\right]
$$

This implies that $a_{33}$ is invertible over $S$. Let

$$
v=\left[\begin{array}{ccc}
1 & 0 & -a_{13} a_{33}^{-1} \\
0 & 1 & -\pi a_{23} a_{33}^{-1} \\
0 & 0 & 1
\end{array}\right], \quad w=\left[\begin{array}{ccc}
1 & 0 & -\pi a_{13} a_{33}^{-1} \\
0 & 1 & -a_{23} a_{33}^{-1} \\
0 & 0 & 1
\end{array}\right]
$$

Then $v, w \in \mathrm{GL}_{n}(S)$ and it is easy to check that

$$
v_{a v}^{*}=\left[\begin{array}{ccc}
x & y & 0 \\
y^{*} & \pi^{2} z & 0 \\
0 & 0 & a_{33}
\end{array}\right], \quad w b w^{*}=\left[\begin{array}{ccc}
\pi^{2} x & y & 0 \\
y^{*} & z & 0 \\
0 & 0 & a_{33}
\end{array}\right] .
$$

where

$$
x=a_{11}-a_{13} a_{33}^{-1} a_{13}^{*}, \quad y=a_{12}-a_{13} a_{33}^{-1} a_{23}^{*} \pi, \quad z=a_{22}-a_{23} a_{33}^{-1} a_{23}^{*} .
$$

Therefore, we may replace $a, b, u$ with $\left[\begin{array}{cc}x & y \\ y^{*} & \pi^{2} z\end{array}\right],\left[\begin{array}{cc}\pi^{2} x & y \\ y^{*} & z\end{array}\right], \pi I_{r} \oplus \pi^{-1} I_{r}$.

Step 4. Using the notation of the previous step, it is left to show that for any *-symmetric $x, z \in \mathrm{M}_{r}(S)$ and $y \in \mathrm{GL}_{r}(S)$, there is $u \in \operatorname{GL}_{n}(S)$ such that $u\left[\begin{array}{cc}x & y \\ y^{*} & \pi^{2} z\end{array}\right] u^{*}=\left[\begin{array}{cc}\pi^{2} x & y \\ y^{*} & z\end{array}\right]$. Replacing $a=\left[\begin{array}{cc}x & y \\ y^{*} & \pi^{2} z\end{array}\right], b=\left[\begin{array}{cc}\pi^{2} x & y \\ y^{*} & z\end{array}\right]$ with $v a v^{*}, v b v^{*}$ for $v=\left[\begin{array}{cc}y^{-1} & 0 \\ 0 & I_{r}\end{array}\right]$, we may further assume that $y=I_{r}$. Assuming this, let

$$
w=(2-\pi x-\pi z)^{-1}
$$

and take

$$
u=\left[\begin{array}{cc}
\pi-2 \pi(1-\pi x) w & 2(1-\pi x) w \\
2(1-\pi z) w & \pi^{-1}-2 \pi^{-1}(1-\pi z) w
\end{array}\right]
$$


(We have $\pi^{-1}-2 \pi^{-1}(1-\pi z) w \in \mathrm{M}_{r}(S)$ since $1-2(1-\pi z) w \equiv 0$ in $S / \pi S$.) The verification of

$$
u\left[\begin{array}{cc}
x & 1 \\
1 & \pi^{2} z
\end{array}\right] u^{*}=\left[\begin{array}{cc}
\pi^{2} x & 1 \\
1 & z
\end{array}\right]
$$

is tedious and left to the reader; the identity $w(2-\pi x-\pi y) w=w$ is used repeatedly to cancel terms.

We now recall the known algorithm to decompose a matrix in $\mathrm{GL}_{n}(K)$ as a product $x u y$ with $x, y \in \mathrm{GL}_{n}(S)$ and diagonal $u \in \mathrm{GL}_{n}(K)$.

Proposition 2. For any $z \in \mathrm{GL}_{n}(K)$, there are $x, y \in \mathrm{GL}_{n}(S)$ and diagonal $u \in \mathrm{GL}_{n}(K)$ with $z=x u y$.

Proof. Write $z=\left(z_{i j}\right)$. Multiplying $z$ on the left and on the right by suitable permutation matrices, we may assume $\nu\left(z_{11}\right)=\min _{i, j} \nu\left(z_{i j}\right)$. We may now replace $z$ with

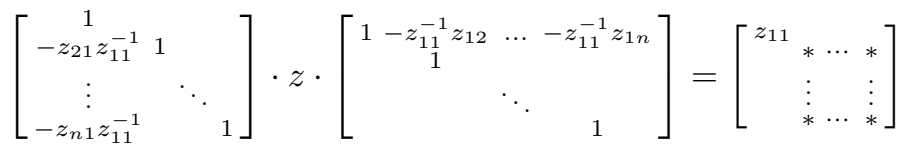

and proceed by induction on $n$.

Remark 3. With some modification, the identity (2) holds even without assuming $K$ is commutative and $\pi$ is central in $K$ (but $\pi^{\sigma}=\pi$ is still assumed). Indeed, if

$$
u=\left[\begin{array}{cc}
\pi-2(1-\pi x)(2-\pi x-\pi z)^{-1} \pi & 2(1-\pi x)(2-\pi x-\pi z)^{-1} \\
2(1-z \pi)(2-x \pi-z \pi)^{-1} & \pi^{-1}-2(1-z \pi)(2-x \pi-z \pi)^{-1} \pi^{-1}
\end{array}\right],
$$

then one has

$$
u\left[\begin{array}{cc}
x & 1 \\
1 & \pi z \pi
\end{array}\right] u^{*}=\left[\begin{array}{cc}
\pi x \pi & 1 \\
1 & z
\end{array}\right] .
$$

Again, the computation is left to the reader.

This modified identity can be used to prove the following stronger version of Theorem 1: Let $K$ be a division ring admitting an additive valuation $\nu: K^{\times} \rightarrow \Gamma$ ( $\Gamma$ is abelian) and an involution $\sigma$ such $\nu(2)=0, \nu \circ \sigma=\nu$, and for all $\gamma \in \Gamma$ there is $a \in K$ with $\nu(a)=\gamma$ and $a=a^{\sigma}$. Let $S:=\{a \in K: \nu(a) \geq 0\}$. Then two unimodular 1-hermitian forms over $(S, \sigma)$ are isometric over $K$ if and only if they are isometric over $S$.

The proof follows the same lines, but requires few additional technicalities, such as verifying that $b^{\prime}=u^{\prime} a u^{\prime}$ is invertible in $\mathrm{M}_{n}(S)$ in Step 2 (the claim $\operatorname{det}\left(b^{\prime}\right)=\operatorname{det}(a) \in S$ is meaningless since $\operatorname{det}(\cdot)$ is no longer defined). This can be settled either by using Dieudonné determinants (together with the easy fact that $S^{\times} /\left[S^{\times}, S^{\times}\right] \hookrightarrow K^{\times} /\left[K^{\times}, K^{\times}\right]$; see [5, p. 64]), or by carefully studying the block form of $a$ and $a^{-1}$ in $\mathrm{M}_{n}\left(S / \pi^{2} S\right)$.

Remark 4. Reversing the proof of Theorem 1 up to Step 2 gives a recipe for producing examples of rationally isometric hermitian forms: Start with a diagonal matrix $u \in \mathrm{GL}_{n}(K)$ such that for every $\gamma \in \Gamma$, the number of diagonal entries with valuation $\gamma$ is the same as the number of diagonal entries with valuation $-\gamma$. Next, choose a matrix $a \in \mathrm{GL}_{n}(S)$ such that $a^{*}=a$ and $b:=u a u^{*} \in \mathrm{GL}_{n}(S)$. The choice of such $a$ can be easily made rigorous. Finally, replace $a, b, u$ with $x a x^{*}$, $y b y^{*}, y u x^{-1}$ for some $x, y \in \mathrm{GL}_{n}(S)$. By the proof of Theorem 1, every example is obtained in this way.

Remark 5. As commented in the beginning, it is possible to derive an algorithm from the proof of Theorem 1. If hermitian forms and isometries are represented as $n \times n$ matrices over $K$, then the operations required are: 
(1) $O(n)$ multiplications and inversions of matrices over $K$ of size at most $n \times n$ (we include here also additions of matrices whose complexity is smaller),

(2) $O\left(n^{3}\right)$ applications of $\nu\left(O(n)\right.$ in Th. 1 and $O\left(n^{3}\right)$ in Pr. 2),

(3) $O\left(n^{3}\right)$ elementary elementary operations $(+,-, \leq)$ in $\Gamma\left(O\left(n^{3}\right)\right.$ comparisons in Pr. 2 and at most $O\left(n^{2}\right)$ operations in Step 2),

(4) $O(n)$ operations of finding perimages for elements of $\Gamma$ in $R$ (Step 2).

Assuming a computational model in which elementary operations in $K$ and $\Gamma$ take $O(1)$ time, the complexity of (a) overtakes all other operations, so this is the asymptotic complexity of the algorithm. A naive implementation of matrix multiplication and inversion would then yield complexity of $O\left(n^{4}\right)$. (More efficient algorithms for matrix multiplication are Strassen and Coppersmith-Winograd, for instance.) There seems be considerable overlap between the matrices being multiplied and inverted through the algorithm, so perhaps further improvement can be achieved by cleverly exploiting this.

In practice, since $K$ is infinite, the complexity of elementary operations in elements of $K$ depends on the number of bits required to represent the elements, and usually these operations yield elements of longer presentation (consider $\mathbb{Q}$ or $\mathbb{F}_{p}\left(t_{1}, \ldots, t_{n}\right)$ for example). Estimating the exact affect of this on the complexity of the algorithm is complicated, and may heavily depend on the base field and its presentation. However, experiments suggests that the practical worst-case complexity is exponential when $K=\mathbb{Q}$; the output isometry has very large nominators and denominators, roughly increasing exponentially in $n$.

A python implementation of the algorithm (which also includes code for producing input for the algorithm) can be found on the author's homepage. It works for valuated involutary division rings as well (implementation of such example is included). Depending on the computer, the worst-case running time for $10 \times 10$ matrices over $\mathbb{Q}$ is about 90 seconds. Of course, a large factor of this can be saved by moving to a lower-level programming language.

Acknowledgements. I deeply thank Eva Bayer-Flückiger for introducing me with the Grothendieck-Serre conjecture, and for hosting me in EPFL during the research.

\section{REFERENCES}

[1] Sofie Beke. Specialisation and good reduction for algebras with involution. preprint, http://www.math.uni-bielefeld.de/lag/man/488.pdf, 2013.

[2] Sofie Beke and Jan Van Geel. An isomorphism problem for azumaya algebras with involution over semilocal bzout domains. Algebras and Representation Theory, pages 1-21, 2014.

[3] Bernhard Keller. A remark on quadratic spaces over noncommutative semilocal rings. Math. Z., 198(1):63-71, 1988.

[4] Manuel Ojanguren and Ivan Panin. Rationally trivial Hermitian spaces are locally trivial. Math. Z., 237(1):181-198, 2001.

[5] Jonathan Rosenberg. Algebraic K-theory and its applications, volume 147 of Graduate Texts in Mathematics. Springer-Verlag, New York, 1994.

[6] Winfried Scharlau. Quadratic and Hermitian forms, volume 270 of Grundlehren der Mathematischen Wissenschaften [Fundamental Principles of Mathematical Sciences]. Springer-Verlag, Berlin, 1985.

Einstein Institute of Mathematics, Hebrew University of Jerusalem

E-mail address: uriya.first@gmail.com 